\title{
Supporting Information. A computational analysis of the ring-opening polymerization of rac-lactide initiated by single-site $\beta$ diketiminate metal complexes: defining the mechanistic pathway and the origin of stereocontrol
}

\section{Vernon C. Gibson, Edward L. Marshall and Henry S. Rzepa}

Department of Chemistry, Imperial College London, SW7 2AY, UK.

\section{Contents}

1. Viewing the Supporting Information

2. Figures $\mathrm{S} 1$ and $\mathrm{S} 2$. Assembly modes considered

3. Table S1. Commentary on the Stationary points on the potential surface for the overall mechanism, illustrated using the RR, SS stereoisomer.

4. Table S2. Commentary on the Four diasteromeric Stationary points corresponding to TS2.

5. Table S3. Commentary on the Four diastereomeric Stationary points corresponding to TS1.

6. ZIP Archive containing these pages for local viewing in a Web browser (open index.html).

\section{Viewing the Supporting Information}

The storyboard below uses the Jmol Java-based applet for visualisation of molecular geometries and animations. To ensure this runs correctly, please install Java JRE Version 1.4.2_5 or higher on your computer, and ensure that the local security policy enables applet display. The coordinates for each computed stationary point are in CML (Chemical Markup Language), MDL Molfile or XYZ animation form.

Key species in this supporting information have had InChI International Chemical identifiers added (in the CML file) to assist in search engine retrieval of structures. See "Enhancement of the Chemical Semantic Web through INChIfication", Simon J. Coles, Nick E. Day, Peter Murray-Rust, Henry S. Rzepa and Yong Zhang, submitted for publication.

\section{Assembly modes considered}

For the insertion of a lactide molecule into the metal-alkoxide bond of a five-membered metallacyclic metal-lactate, there are a total of eight possible assembly modes. Both the approaching monomer, and the two chiral centres of the last inserted repeat unit, may be either $(\mathrm{R}, \mathrm{R})$ or $(\mathrm{S}, \mathrm{S})$. Further more, the incoming monomer may approach either face of the metal-lactate chelate. This is illustrated below in Fig. 1S for the RR,SS mode of addition described in the accompanying communication, with attack from above the metal-lactate, and for the approach of an $(\mathrm{R}, \mathrm{R})$-lactide molecule from below the lactate chelate formed from the ring-opening of $(\mathrm{S}, \mathrm{S})$-lactide. These are enantiomers and will therefore possess the same energy. Fig. $2 \mathrm{~S}$ schematically represents all eight assembly modes, shown in their four enantiomeric pairs.
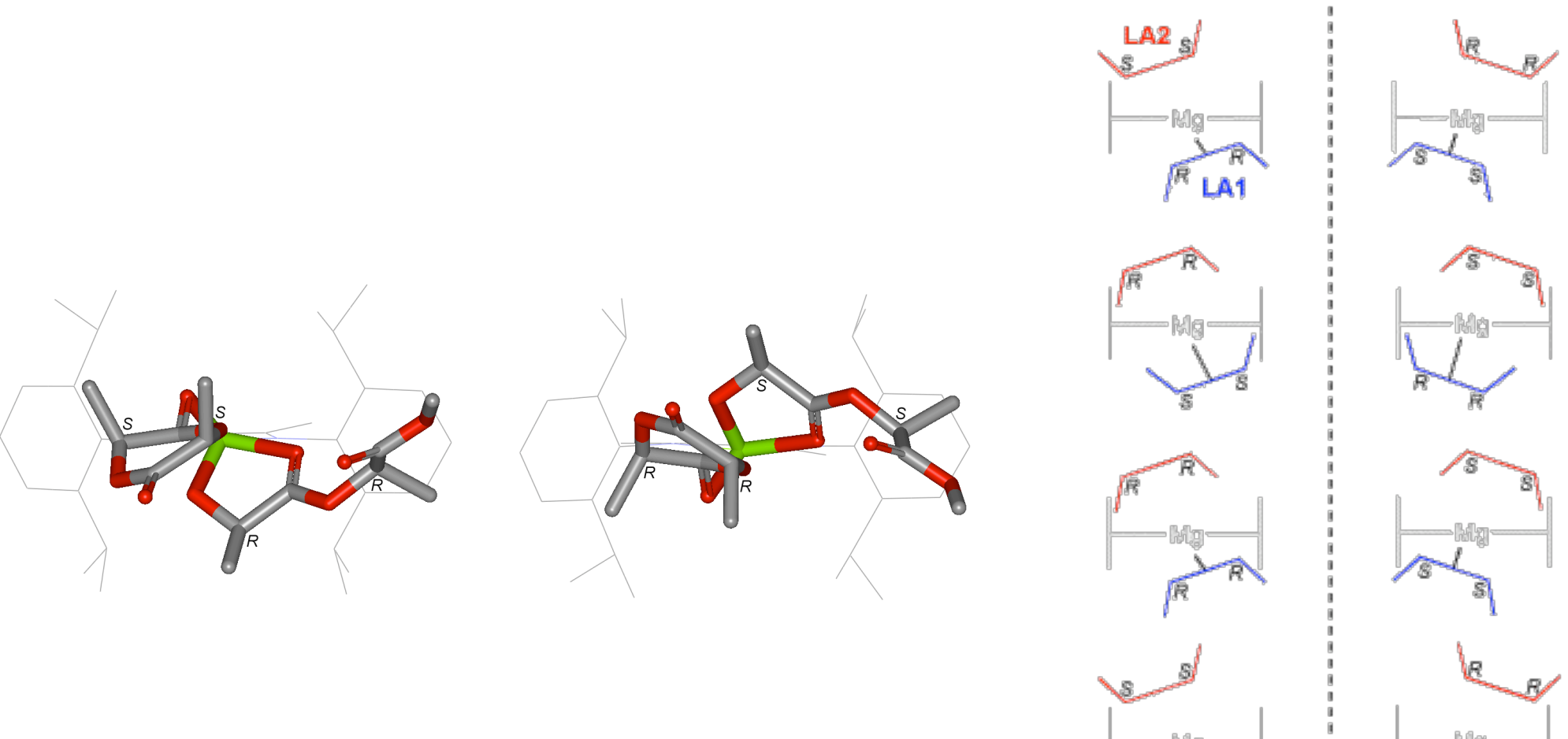


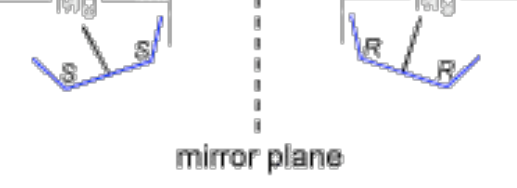

Figure 1S. An enantiomeric pair of assembly modes

Figure 2S. The eight assembly modes divided into four enantiomeric pairs: the four alternatives in the left hand column are those described in the communication (from top to bottom: RR,SS; SS,RR; $\mathrm{RR}, \mathrm{RR} ; \mathrm{SS}, \mathrm{SS}$ ).

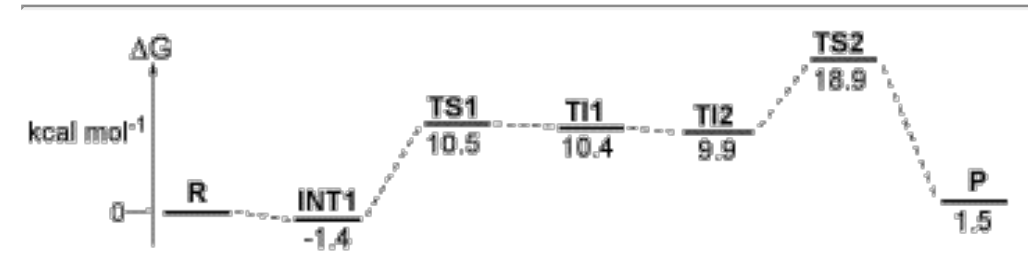

